\title{
Oclusão Percutânea do Pequeno Canal Arterial com Molas de Gianturco: Impacto da Otimização da Seleção das Molas e dos Pacientes e da Não-Tolerância ao Fluxo Residual Significativo Imediato nos Resultados
}

\author{
Carlos Augusto Cardoso Pedra ${ }^{1,2}$, César Augusto Esteves ${ }^{1}$, Sérgio Luiz Navarro Braga ${ }^{1}$, \\ Simone Rolim Fernandes Fontes Pedra ${ }^{1,2}$, Sérgio Cunha Pontes Júnior ${ }^{1}$, \\ Maria Aparecida de Paula Silva', Gisele de Montalvão e Alpuim Lousadas Almeida', \\ Maria Virgínia Tavares Santana1, Valmir Fernandes Fontes ${ }^{1,2}$
}

\section{RESUMO}

Introdução: A oclusão percutânea do canal arterial persistente (PCA) com implante retrógrado não controlado de molas de Gianturco tem sido freqüentemente empregada. Método: Desde janeiro de 2001, temos aplicado essa técnica em pacientes com canais $<3 \mathrm{~mm}$, dos tipos A, D e E, e não tolerado fluxos residuais imediatos significativos, implantando outra mola. Neste artigo, descrevemos os resultados desse tipo de abordagem. Resultados: Desde janeiro de 2001, 178 pacientes (110 do sexo feminino: mediana de idade e peso: cinco anos e $17 \mathrm{~kg}$, respectivamente) foram submetidos ao procedimento. A média do diâmetro mínimo do PCA foi de 1,8 $\pm 0,6 \mathrm{~mm}(0,5 \mathrm{~mm}$ a 3,0 mm), sendo 157 do tipo $A$, nove do tipo $D$ e 12 do tipo E. Em três pacientes, houve embolização inicial das molas com resgate, sendo reimplantadas em dois (taxa de sucesso: 99,4\%). Mais de uma mola foi utilizada em $32(18 \%)$ pacientes, com canais significativamente maiores que os restantes $(2,3 \pm 0,5 \mathrm{~mm}$ vs. $1,6 \pm 0,5 \mathrm{~mm}$; $p<0,001)$. Oclusão imediata foi observada em 160 pacientes e $17(9,6 \%)$ possuíam fluxo residual discreto, difuso e de baixa velocidade. Redução de pulsos e transfusão foram complicações observadas em dois pacientes. Um paciente com fluxo residual discreto não retornou. Todos os 176 pacientes, à ecocardiografia, apresentavam oclusão e nenhum apresentava distúrbios de fluxo na aorta ou na artéria pulmonar no seguimento (mediana: seis meses). Conclusão: A tolerância zero para fluxos residuais imediatos e a seleção otimizada das molas e dos

\section{SUMMARY}

Percutaneous Closure of the Small Patent Ductus Arteriosus with Gianturco Coils. Impact of the Optimal Patient and Coil Selection and the No Tolerance Policy for Significant Immediate Residual Leaks on Outcomes

Introduction: Percutaneous occlusion of the patent ductus arteriosus (PDA) by non-controlled retrogade release of Gianturco coils has been frequently employed. Methods: Since January of 2001, we have applied this technique for patients with type A, D and E PDAs with $<3 \mathrm{~mm}$ and have not tolerated significant immediate residual leaks, implanting additional coils. In this paper we report the outcome of this approach. Results: From January 2001, 178 patients (110 females; median age and weight: 5 years and $17 \mathrm{~kg}$, respectively) underwent the procedure. The mean minimal diameter of the PDA was $1.8 \pm 0.6 \mathrm{~mm}(0.5$ to $3.0 \mathrm{~mm})$ with 157,9 and 12 being of types $A, D$ and $E$, respectively. In 3 patients, there was initial embolization with percutaneous retrieval followed by coil re-implantation in 2 (success rate: 99.4\%). More than one coil was required in 32 patients with PDAs significantly larger than the remainder $(2.3 \pm 0.5$ versus $1.6 \pm 0.5 \mathrm{~mm} ; \mathrm{p}<0.001)$. Immediate occlusion was observed in 160 patients with 17 (9.6\%) having discrete, diffuse, low velocity residual leaks. Pulse reduction and transfusion were complications observed in 2 patients. One patient with mild residual leak was lost to

\footnotetext{
Instituto Dante Pazzanese de Cardiologia - São Paulo, SP.

2 Hospital do Coração - Associação do Sanatório Sírio - São Paulo, SP. Correspondência: Carlos A. C. Pedra. Instituto Dante Pazzanese de Cardiologia. Av. Dr. Dante Pazzanese, 500 - 14o andar - Ibirapuera - São Paulo, SP - CEP 04012-909.

Tel.: (11) 5085-6114 - Fax: (11) 5085-6196 •E-mail: cacpedra@uol.com.br Recebido em: 21/12/2007 • Aceito em: 31/1/2008
} 
Pedra CAC, et al. Oclusão Percutânea do Pequeno Canal Arterial com Molas de Gianturco: Impacto da Otimização da Seleção das Molas e dos Pacientes e da Não-Tolerância ao Fluxo Residual Significativo Imediato nos Resultados. Rev Bras Cardiol Invas. $2008 ; 16(1): 86-90$.

pacientes, provavelmente, explicam esses ótimos resultados. O procedimento é simples, custo efetivo, seguro e altamente eficaz.

DESCRITORES: Persistência do conduto arterioso. Cateterismo cardíaco. Próteses e implantes. Embolização terapêutica.

A oclusão percutânea do canal arterial é um dos procedimentos intervencionistas para cardiopatias congênitas mais freqüentemente realizados no Laboratório de Cateterismo. Desde sua descrição por Cambier et al. ${ }^{1}$, em 1992, o fechamento com coils de Gianturco pela via retrógrada clássica tem se mostrado uma técnica segura, barata e eficiente para pequenos canais medidos pela angiografia ${ }^{1,2}$. Entretanto, maiores índices de insucesso no implante, de mau posicionamento final da mola, de embolização, de fluxos residuais, de hemólise e de obstrução parcial do fluxo para a artéria pulmonar esquerda ou aorta têm sido observados em canais maiores que 3,0 mm, dos tipos B e C e/ou quando a seleção das molas é inapropriada ${ }^{3-7}$. Nosso grupo postulou que tais ocorrências seriam provavelmente minimizadas com a otimização da seleção dos dispositivos e dos pacientes. Neste estudo, descrevemos nossa experiência com esse tipo de abordagem otimizada para o fechamento do canal arterial persistente (PCA) em um hospital de ensino.

\section{MÉTODO}

\section{Seleção dos pacientes}

Todos os pacientes com diagnóstico clínico e ecocardiográfico de PCA nativo e restritivo, com fluxo da esquerda para a direita e com peso maior que $5 \mathrm{~kg}$ eram candidatos ao implante. O diagnóstico de PCA restritivo foi definido pela ausência clínica de insuficiência cardíaca, pouco ou nenhum aumento da área cardíaca na radiografia de tórax, pouca ou nenhuma sobrecarga ventricular esquerda à eletrocardiografia e presença de gradientes elevados entre a aorta e a artéria pulmonar à ecocardiografia. A partir de janeiro de 2001, optamos por aplicar a técnica de implante descrita a seguir somente para os pacientes portadores de canais dos tipos A, D e E, segundo a classificação angiográfica de Krichenko et al. ${ }^{8}$ e com diâmetro mínimo menor ou igual a $3 \mathrm{~mm}$ à angiografia em perfil esquerdo. Não foram excluídos os canais "silenciosos", sem repercussão hemodinâmica, encaminhados para oclusão pelo médico de referência. Excluíram-se os pacientes com peso inferior a $5 \mathrm{~kg}$, os com fluxo follow up. On echocardiography, all 176 patients had occlusion and none had flow disturbances in the aorta or in the pulmonary artery on follow up (average 6 months). Conclusion: Zero tolerance for significant immediate residual leaks and optimized selection of coils and patients probably explain these excellent outcomes. The procedure is easy to perform, cost effective, safe and highly efficient.

DESCRIPTORS: Ductus arteriosus, patent. Heart catheterization. Prostheses and implants. Embolization, therapeutic.

residual após implante prévio de outro dispositivo, os com hiper-resistência vascular pulmonar fixa e aqueles portadores de canais dos tipos B e C da classificação angiográfica de Krishenko et al. ${ }^{8}$ e/ou com diâmetro mínimo maior que $3 \mathrm{~mm}$.

\section{Técnica e seleção das molas}

Sob anestesia geral e intubação orotraqueal ou sedação consciente em pacientes maiores, puncionouse a artéria femoral com introdução de bainhas $4 \mathrm{~F}$ a $6 \mathrm{~F}$, dependendo do tamanho do paciente. Administrou-se heparina (100-150 U/kg, com dose máxima de $5.000 \mathrm{U})$ e cefazolina $(30 \mathrm{mg} / \mathrm{kg})$. Pela via arterial, em perfil esquerdo, realizou-se aortografia no canal arterial com cateter pig-tail para aferição de seu diâmetro mínimo, comprimento e dimensões da ampola aórtica. Com tais dados, selecionou-se a mola de forma que o diâmetro de sua alça fosse pelo menos duas vezes maior que o diâmetro mínimo do canal, com comprimento suficiente para a formação de três a cinco alças, assegurando-se que as dimensões da ampola aórtica acomodariam as dimensões da alça da mola do lado aórtico. Além disso, optou-se pela seleção de molas com menor número de alças (três ou quatro) nos casos de canais mais curtos e/ou com ampola rasa. Por outro lado, em canais com mais de $2,5 \mathrm{~mm}$ de diâmetro mínimo e/ou naqueles de alto fluxo, deu-se preferência ao uso de molas com, no mínimo, quatro alças. Em todos os casos, utilizaram-se molas de 0,038 polegada de espessura (diâmetro interno), por ter maior poder de retração elástica e compactação que a de 0,035 polegada. A partir de janeiro de 2001, sete tipos de molas ficaram permanentemente disponíveis para uso rotineiro em nossa instituição e foram utilizadas neste estudo, a saber: $38-4-3$; 38-5-4; 38-5-5; 38-85 ; 38-8-6; 38-8-8; e 38-10-8. O primeiro número se refere à espessura da mola (diâmetro interno) em centésimos de polegada; o segundo número, ao comprimento em centímetros; e o terceiro número, ao diâmetro da alça em milímetros.

Atravessou-se o canal arterial pelo lado aórtico com o auxílio de guia hidrofílico e progrediu-se um cateter Judkins de artéria coronária direita para a arté- 
Pedra CAC, et al. Oclusão Percutânea do Pequeno Canal Arterial com Molas de Gianturco: Impacto da Otimização da Seleção das Molas e dos Pacientes e da Não-Tolerância ao Fluxo Residual Significativo Imediato nos Resultados. Rev Bras Cardiol Invas. $2008 ; 16(1): 86-90$.

ria pulmonar, permitindo a mensuração das pressões na artéria pulmonar. Transferiu-se a mola selecionada do carregador metálico para o cateter Judkins, empurrando-se a ponta dura de um guia de 0,035 polegada ou 0,038 polegada. Após a mola ter sido avançada por cerca de $20 \mathrm{~cm}$ a $30 \mathrm{~cm}$ dentro do cateter, empurrou-se a mesma utilizando-se a ponta mole, reta ou retificada, do mesmo guia. Empurrou-se o dispositivo até que fosse exteriorizado gradativamente na artéria pulmonar, permitindo a formação de cerca de uma alça. Nesse momento, puxou-se todo o sistema para a aorta como uma unidade até a extremidade pulmonar da mola ancorar, no ponto mais estreito do canal e previamente definido pela angiografia, levando-se em conta a sombra traqueal para referência. Nesse momento, manteve-se o guia em posição fixa e puxou-se o cateter sobre o mesmo para expor o restante da mola na aorta. Dessa forma, as alças do lado esquerdo foram formadas e acomodadas na ampola aórtica após a mola sair do cateter, levando-se em conta seu poder de retração elástica. Na maioria dos casos, o médico residente em treinamento em Cardiologia Intervencionista realizou o implante, sendo supervisionado por um dos autores responsáveis pelo Laboratório de Cateterismo. A critério do operador responsável, aplicaramse algumas variações técnicas no procedimento de implante. Em alguns casos de canais com mais de 2,5 $\mathrm{mm}$ e de alto fluxo, empregou-se a técnica já descrita de implante com auxílio de um cateter balonado posicionado por via anterógrada ${ }^{9}$.

Após o implante, foram realizadas aortografias de controle com pequenas injeções de contraste pelo cateter Judkins, com o intuito de averiguar o posicionamento do dispositivo. Cerca de cinco minutos após a liberação final do cateter, praticou-se nova angiografia com cateter pig-tail, para avaliar a presença e a magnitude de possíveis fluxos residuais. Aceitaram-se fluxos residuais discretos, difusos, de baixa velocidade, acreditando-se na oclusão espontânea dos mesmos com o tempo. Na presença de fluxos residuais bem definidos, tipo em jato, cruzou-se o canal novamente por via retrógrada para implante de novo coil. Em casos de mau posicionamento ou de embolização, puncionou-se a veia femoral para resgate da mola com cateteres tipo laço. Após o término das manipulações com cateteres, reverteu-se cerca de 50\% da dose de heparina com protamina e obteve-se hemostasia por meio de compressão manual. Procedeu-se à extubação dentro do Laboratório de Cateterismo e realizou-se a monitoração rotineira na unidade de recuperação anestésica.

\section{Seguimento}

Além da avaliação clínica, foram realizados uma radiografia de tórax, um eletrocardiograma e um ecocardiograma transtorácico no mesmo dia da alta hospitalar ou nas primeiras 72 horas seguintes ao procedimento. Retornos foram agendados aos seis e aos 12 meses após o procedimento, para reavaliação tanto clínica como ecocardiográfica. Os pacientes com oclusão completa após seguimento de um ano receberam alta da instituição. Nos casos de fluxo residual persistente, considerava-se um novo procedimento percutâneo para tentar oclusão do canal arterial um ano após o procedimento inicial ${ }^{10}$.

\section{Análise estatística}

Os valores são expressos em médias e desvio padrão ou medianas e variação, conforme indicado. A comparação de médias foi realizada aplicando-se o teste $t$ de Student e um valor de $p<0,05$ foi considerado estatisticamente significante.

\section{RESULTADOS}

\section{População}

No período de janeiro de 2001 a dezembro de 2007, 178 pacientes selecionados com PCA nativo (110 do sexo feminino) foram submetidos ao procedimento. O peso dos pacientes variou entre $8 \mathrm{~kg}$ e 80 $\mathrm{kg}$ (mediana $=17 \mathrm{~kg}$ ) e a idade, de oito meses a 60 anos (mediana $=$ cinco anos). Cinco pacientes eram portadores de quadros sindrômicos (Down, Rubstein, rubéola congênita); três, de valva aórtica bicúspide; dois, de comunicação interventricular (CIV) muscular de $1 \mathrm{~mm}$; e um, de estenose discreta na artéria pulmonar esquerda. Um paciente tinha sido operado de defeito do septo atrioventricular anos antes do procedimento.

\section{Características do canal arterial}

Entre os pacientes avaliados, 157 (88\%) possuíam canal arterial do tipo A, nove (5\%) do tipo D e 12 (7\%) do tipo E. O diâmetro mínimo do PCA variou de $0,5 \mathrm{~mm}$ a $3,0 \mathrm{~mm}$ (média $=1,8 \pm 0,6 \mathrm{~mm}$ ). Nenhum paciente possuía pressão arterial sistólica na artéria pulmonar maior que $35 \mathrm{mmHg}$ e pressão média maior que $25 \mathrm{mmHg}$.

\section{Resultados imediatos}

Em três pacientes, houve embolização inicial dos dispositivos com resgate imediato. Molas de diâmetro externo maior foram reimplantadas com sucesso em dois pacientes, perfazendo taxa de sucesso no implante de 99,4\%. O paciente que não apresentou sucesso tinha um ano de idade $(9 \mathrm{~kg})$ e possuía canal do tipo A, com 2,5 mm e alto fluxo. Tentou-se implantar dois coils (38-8-5 e 38-5-5) sem sucesso, sendo abortado o procedimento. Em novo procedimento, seis meses mais tarde, o canal mediu 3,3 mm e foi ocluído com prótese Amplatzer (AGA) 8-6, segundo técnicas já descritas ${ }^{11-13}$. Houve necessidade de mais de uma mola para oclusão do PCA no mesmo procedimento em 32 (18\%) pacientes, em decorrência de fluxos residuais iniciais significativos. A média do diâmetro 
Pedra CAC, et al. Oclusão Percutânea do Pequeno Canal Arterial com Molas de Gianturco: Impacto da Otimização da Seleção das Molas e dos Pacientes e da Não-Tolerância ao Fluxo Residual Significativo Imediato nos Resultados. Rev Bras Cardiol Invas. $2008 ; 16(1): 86-90$.

mínimo do PCA nos pacientes que receberam mais de uma mola foi significativamente maior que naqueles que receberam uma mola apenas $(2,3 \pm 0,5 \mathrm{~mm}$ vs. 1,6 $\pm 0,5 \mathrm{~mm} ; \mathrm{p}<0,001)$. Oclusão completa imediata foi observada em $160(90,4 \%)$ pacientes e 17 (9,6\%) pacientes saíram da sala de cateterismo com fluxo residual discreto, difuso e de baixa velocidade. Ausência temporária de pulsos em membros inferiores foi observada em dois pacientes e outros dois necessitaram transfusão de sangue. Todos tiveram alta no mesmo dia ou no dia seguinte ao procedimento.

\section{Resultados de seguimento}

Um paciente de um ano de idade, com PCA de $3,0 \mathrm{~mm}$ do tipo A e fluxo residual discreto após implante de seis molas, não voltou para acompanhamento e realização de exames de controle. Nos 176 pacientes restantes com pelo menos um retorno ambulatorial para realização de ecocardiografia, observou-se oclusão total do PCA em todos (seguimento mediano de seis meses). Em nenhum dos casos houve embolização tardia do dispositivo, endocardite ou aumento da velocidade de fluxo na artéria pulmonar esquerda e na aorta nas ecocardiografias de controle. Nenhum paciente foi submetido a procedimento de reoclusão com implante de nova mola.

\section{DISCUSSÃO}

A oclusão percutânea do canal arterial com coils de Gianturco é um procedimento simples e de excelente custo-benefício, tendo sido empregado como técnica de escolha para o PCA de pequenas dimensões na maioria dos centros mundiais. Entretanto, os resultados apresentados neste estudo foram sensivelmente melhores que os já publicados pelo nosso grupo, sendo provavelmente decorrentes de aprendizagem e aperfeiçoamento contínuos. Este trabalho demonstrou que a otimização da seleção das molas e dos pacientes e uma postura de não tolerância a fluxos residuais imediatos significativos tiveram grande impacto nos desfechos finais quase que ideais aqui observados. Entretanto, para a aplicação dessa abordagem, reconhecemos que as molas utilizadas neste estudo devem estar sempre disponíveis, o que nem sempre é possível em nosso meio. A mola 38-8-6, por exemplo, foi especialmente encomendada e manufaturada pela fábrica nos Estados Unidos para abordagem de canais entre $2,5 \mathrm{~mm}$ e $3,0 \mathrm{~mm}$ de diâmetro mínimo. Em canais dos tipos $\mathrm{B}$ e $\mathrm{C}$ e/ou nos maiores que $3 \mathrm{~mm}$, a oclusão com molas de Gianturco de liberação nãocontrolada não deve ser utilizada, por apresentar maiores dificuldades técnicas e por se associar a maiores taxas de insucesso, mau posicionamento, embolização, fluxos residuais com hemólise e necessidade de uso de múltiplos coils, resultando em obstruções parciais de fluxo para a artéria pulmonar esquerda e aorta em pequenos lactentes, como foi demonstrado na literatura $^{3-7}$. Nesta casuística, o único caso de insucesso foi observado em canal do tipo A, que possuía medidas significativamente maiores em um segundo cateterismo $(2,5 \mathrm{~mm}$ vs. $3,3 \mathrm{~mm})$. Essa variação na medida do diâmetro mínimo, provavelmente, reflete a natureza dinâmica do canal pela ocorrência de espasmos. Apesar de essa observação ser vivenciada freqüentemente na prática clínica, é difícil o encontro de evidências científicas na literatura que comprovem tal hipótese. Nos casos de PCAs maiores que 3,0 mm, técnicas alternativas para implante de molas mais espessas ${ }^{14-19}$ ou o emprego de outras próteses, como Amplatzer ${ }^{11-13}$ e Nit-Occlud ${ }^{20}$, são opções mais seguras e eficazes.

Do ponto de vista técnico, a simplicidade do procedimento permite até que o mesmo seja realizado por intervencionistas em fase (adiantada) de treinamento, sem que haja comprometimento dos resultados. Apesar de a liberação da mola ser classicamente considerada como "não-controlada", tal denominação, em nossa opinião, deve ser revista. O operador tem total capacidade de controlar o correto posicionamento do dispositivo se os passos técnicos do implante forem seguidos sistematicamente. O desfecho ideal parece estar mais associado à escolha adequada da mola para o tipo do canal estudado que à própria capacidade e habilidade técnica do operador, como demonstrou este estudo. É claro que a técnica de implante aqui descrita dá menos margem a possíveis erros, que seriam minimizados com um sistema controlado de implante $^{21}$. Com esses sistemas, se o implante não for satisfatório, o posicionamento do dispositivo pode ser refeito sem maiores dificuldades, apenas puxando a mola para dentro do cateter e reposicionando-a. Entretanto, tais sistemas também podem depender de uma curva de aprendizado inicial para domínio da técnica, além de serem mais caros. Ainda em relação aos aspectos técnicos, é preferível o uso de coils de 0,038 polegada aos de 0,035 polegada, pelo fato de estes apresentarem maior perfil, robustez, força radial e superfície de contato, permitindo melhor compactação dentro da ampola. A nosso ver, as molas de 0,035 polegada poderiam ser excepcionalmente utilizadas em canais com cerca de $1 \mathrm{~mm}$ de diâmetro. Outro ponto que merece comentários é a preferência pelo uso de molas com alça de diâmetro apropriado para se acomodar dentro da ampola aórtica, nos casos de canais dos tipos A e E. Molas com diâmetro excessivamente grande para o diâmetro da ampola, além de serem provavelmente desnecessárias para a oclusão, podem ser teoricamente deletérias na medida em que sofrem protrusão para o lúmen aórtico, dificultando a endotelização e/ou levando ao aparecimento de gradientes locais, especialmente em lactentes. O comprimento do canal arterial também é um parâmetro que deve ser levado em conta para escolha correta do dispositivo. Canais mais curtos e/ou com ampola rasa devem ser ocluídos com molas com menor número de alças (três ou quatro) para evitar possíveis protrusões, como já foi mencionado. Por outro lado, canais mais 
Pedra CAC, et al. Oclusão Percutânea do Pequeno Canal Arterial com Molas de Gianturco: Impacto da Otimização da Seleção das Molas e dos Pacientes e da Não-Tolerância ao Fluxo Residual Significativo Imediato nos Resultados. Rev Bras Cardiol Invas. $2008 ; 16(1): 86-90$.

longos podem ser ocluídos com molas de até cinco alças, viabilizando a presença de maior número de alças dentro da ampola ductal, o que provavelmente aumenta as chances de oclusão.

Apesar de tentarmos, sempre que possível, utilizar um único coil para o fechamento dos defeitos, notamos que em 32 (18\%) pacientes houve necessidade do uso de múltiplos dispositivos. Tais pacientes eram portadores de canais de alto fluxo e maiores que o restante da casuística (geralmente maiores que $2 \mathrm{~mm}$ ). A presença de tais características deve alertar o operador quanto à necessidade do uso de mais de um dispositivo. Acreditamos que essa postura de não tolerar a presença de fluxos residuais bem definidos do tipo em jato após o implante da primeira mola contribuiu muito para os excelentes índices de oclusão aqui observados. Por outro lado, como nossa casuística era composta de pacientes com mais de $8 \mathrm{~kg}$ de peso e como o posicionamento das próteses foi considerado ótimo nesses pacientes com mais de um dispositivo, não se observaram obstruções parciais de fluxo na artéria pulmonar e na aorta. O fato de nenhum dos pacientes com fluxo residual imediato discreto ter necessitado de procedimento de reoclusão no seguimento mostra que a decisão de se tolerar fluxos residuais imediatos difusos e de baixa velocidade, com aspecto em "fumaça", parece ser apropriada, como já foi demonstrado em experiências anteriores ${ }^{14}$.

Apesar de demonstrar excelentes resultados que parecem ser melhores que os observados no passa$\mathrm{do}^{4}$, este estudo apresenta uma limitação metodológica, que é a falta de um grupo controle. No entanto, em nossa opinião, essa limitação não denigre a mensagem central que queremos transmitir.

\section{CONCLUSÕES}

A oclusão do PCA restritivo $\leq 3 \mathrm{~mm}$ dos tipos $A$, $\mathrm{D}$ e $\mathrm{E}$ por meio da técnica de liberação não-controlada por via retrógrada de uma ou mais molas de Gianturco é de simples realização, de custo limitado, segura e altamente eficaz. Constitui-se, provavelmente, na melhor opção terapêutica disponível nos dias de hoje.

\section{REFERÊNCIAS BIBLIOGRÁFICAS}

1. Cambier PA, Kirby WC, Wortham DC, Moore JW. Percutaneous closure of the small (less than $2.5 \mathrm{~mm}$ ) patent ductus arteriosus using coil embolization. Am J Cardiol. 1992; 69(8):815-6.

2. Hijazi ZM, Geggel RL. Results of anterograde transcatheter closure of patent ductus arteriosus using single or multiple Gianturco coils. Am J Cardiol. 1994;74(9):925-9.

3. Hijazi ZM, Geggel RL. Transcatheter closure of large patent ductus arteriosus ( $>$ or $=4 \mathrm{~mm}$ ) with multiple Gianturco coils: immediate and mid-term results. Heart. 1996;76(6):536-40.

4. Pedra CA, Pedra SR, Esteves CA, Braga SL, Silva MA, de Almeida TL, et al. Overall experience with percutaneous occlusion of patent ductus arteriosus. Arq Bras Cardiol. 1998;71(6):769-80.
5. Moore JD, Shim D, Mendelsohn AM, Kimball TR. Coarctation of the aorta following coil occlusion of a patent ductus arteriosus. Cathet Cardiovasc Diagn. 1998;43(1):60-2.

6. Tomita H, Fuse S, Akagi T, Matsumoto Y, Murakami Y, Shiraya $\mathrm{H}$, et al. Hemolysis complicating coil occlusion of the patent ductus arteriosus. Cathet Cardiovasc Diagn. 1998; 43(1):50-3.

7. Carey LM, Vermilion RP, Shim D, Lloyd TT, Beeekman RH $3^{\text {rd }}$, Ludomirsky A. Pulmonary artery size and and flow disturbances after patent ductus arteriosus coil occlusion. Am J Cardiol. 1996;78(11):1307-10.

8. Krichenko A, Benson LN, Burrows P, Möes CA, McLaughlin $\mathrm{P}$, Freedom RM. Agiographic classification of the isolated, persistently patent ductus arteriosus and implications for percutaneous catheter oclusion. Am J Cardiol. 1989;63(12): 877-80.

9. Esteves CA, Braga SL, Pedra CA, Fontes VF. Implante assistido de mola de Gianturco no canal arterial através da ajuda de cateter balonado. Rev Bras Cardiol Invas. 1997;5(3):6-11.

10. Pedra CA, Esteves CA, Pedra SR, Braga SL, Sousa JE, Fontes $V F$. Indications, technique, results and clinical impact of reocclusion procedures for residual shunts after transcatheter closure of patent ductus arteriosus. Arch Inst Cardiol Mex. 1999;69(4):320-9.

11. Faella $\mathrm{H}$, Hijazi ZM. Closure of the patent ductus arteriosus with the Amplatzer PDA device: immediate results of the international clinical trial. Catheter Cardiovasc Interv. 2000; 51(1):50-4.

12. Simões LC, Pedra CA, Esteves CA, Camargo R, Braga SL, Loureiro $\mathrm{P}$, et al. Percutaneous closure of ductus arteriosus with the Amplatzer prosthesis. The Brazilian experience. Arq Bras Cardiol. 2001;77(6):520-31.

13. Chamie F, Chamie D, Ramos S. Oclusão percutânea dos canais arteriais com prótese Amplatzer. Rev Bras Cardiol Invas. 2007;15(1):15-24.

14. Arnoni DG, Pena JJS, Fontes VF, Braga SLN, Esteves CA, Ferreira WP, et al. Oclusão percutânea do canal arterial > $3 \mathrm{~mm}$ com auxílio do biótomo. Rev Bras Cardiol Invas. $2007 ; 15(2): 134-40$.

15. Hays MD, Hoyer MH, Glasow PF. New forceps delivery technique for coil occlusion of patent ductus arteriosus. Am J Cardiol. 1996;77(2):209-11.

16. Hays MD. Anterograde coil closure of patent ductus arteriosus using a modified bioptome delivery technique. Catheter Cardiovasc Interv. 2000;50(2):191-4.

17. Grifka RG, Jones TK. Transcatheter closure of large PDA using 0.052" Gianturco coils: controlled delivery using a bioptome catheter through a 4 French sheath. Cathet Cardiovasc Interv. 2000;49(3):301-6.

18. Kumar RK, Krishnan MN, Venugopal K, Sivakumar K, Anil SR. Bioptome-assisted simultaneous delivery of multiple coils for occlusion of the large patent ductus arteriosus. Catheter Cardiovasc Interv. 2001;54(1):95-100.

19. Kumar RK, Anil SR, Kannan BR, Philip A, Sivakumar K. Bioptome-assisted coil occlusion of moderate-large patent ductus arteriosus in infants and small children. Catheter Cardiovasc Interv. 2004;62(2):266-71.

20. Moore J, DiMeglio D, Javois AP, Takahashi M, Berdjis F, Cheatham JP. Results of the phase I food and drug administration clinical trial of duct-occlud device occlusion of patent ductus arteriosus. Catheter Cardiovasc Interv. 2001; 52(1):74-8.

21. Tometzki AJ, Arnold R, Peart I, Sreeram N, Abdulhamed JM, Godman MJ, et al. Transcatheter occlusion of the patent ductus arteriosus with Cook detachable coils. Heart. 1996; 76(6):531-5. 